\title{
ON NON-ISOTROPIC HOMOGENEOUS LIPSCHITZ SPACES
}

\author{
STEFANO MEDA
}

(Received 14 September 1987)

Communicated by W. Moran

\begin{abstract}
We prove that in a non-isotropic Euclidean space, homogeneous Lipschitz spaces of distributions, defined in terms of (generalized) Weierstrass integrals, can be characterized by means of higher order difference operators.
\end{abstract}

1980 Mathematics subject classification (Amer. Math. Soc.) (1985 Revision): 58 O 50; secondary 42 B 99.

Keywords and phrases: Lipschitz spaces, higher order differences, Weierstrass kernel.

Homogeneous Lipschitz classes on the $n$-dimensional Euclidean space were first introduced and extensively studied by C. Herz (see [8]); he defined them by means of higher order difference operators.

Another approach had previously been developed by M. H. Taibleson (see [18], [19] and, for further results in this direction, [2] and [13]), who defined non-homogeneous Lipschitz spaces in terms of Weierstrass integrals.

The main purpose of this paper is to prove that, in the homogeneous case, these two points of view give rise to equivalent spaces of non-isotropic $\mathbf{R}^{n}$.

To do this we need non-isotropic higher order difference operators and a substitute for the Weierstrass kernel. Non-isotropic difference operators were introduced by Soardi in [15]; he also defined the homogeneous Lipschitz spaces $\Lambda_{k, \alpha}^{\infty, \infty}$ (in our notation) and studied their connection with some Morrey-Campanato spaces (see also [12] for related results).

(C) 1989 Australian Mathematical Society $0263-6115 / 89 \$ A 2.00+0.00$ 
A fundamental solution of a suitable hypoelliptic differential operator provides a kernel with the required properties in the case when the dilations are diagonal and with rational eigenvalues (see [4] for related kernels on nilpotent groups).

Non-isotropic Lipschitz spaces had previously been studied also by A. Torchinski (see [20]) from a very general point of view. However he considered non-homogeneous spaces and his treatment did not cover the case $p=1$; moreover his methods are considerably less direct and elementary than ours. For some related results and material about Lipschitz spaces see also [10] (on the Heisenberg group), [3], [4] and [6] (on stratified groups) and [11], [14] (in a very general context).

Our paper is organized as follows.

In Section 1 we define $\Lambda_{k, \alpha}^{p, q}$ to be the space of tempered distributions whose generalized Weierstrass integral satisfies a suitable growth condition and prove that $\Lambda_{k, \alpha}^{p, q} / \wp^{k}$ are equivalent provided $k>\alpha$. Note that our proof is elementary and does not involve the theory of the Morrey-Campanato spaces. As a consequence of the techniques developed, we obtain a useful characterization of Lipschitz spaces and we give an application to non-isotropic Bessel potential operators.

In Section 2 we prove our main result, the equivalence between Lipschitz spaces defined in terms of (generalized) Weierstrass integrals and in terms of higher order difference operators.

I wish to thank Professor P. M. Soardi for many valuable conversations about the subject of this paper.

\section{Notation}

Suppose that $\left\{A_{t}\right\}_{l>0}$ is a group of linear transformations $R^{n}$ of the form $A_{t}=\operatorname{diag}\left(t^{\rho_{1}}, \ldots, t^{\rho_{n}}\right), \rho_{j} \geq 1$ rational, $j=1, \ldots, n$.

We set $\rho=\left(\rho_{1}, \ldots, \rho_{n}\right)$, and $Q=\rho_{1}+\cdots+\rho_{n}$ is the homogeneous dimension of $R^{n}$. We shall write $t x$ instead of $A_{t} x$. We use standard notation for multi-index; $|I|$ will denote the length of $I$ and its homogeneous length is defined as $d(I)=\rho_{1} i_{1}+\cdots+\rho_{n} i_{n}$. The set of the numbers of the form $d(I)$, when $I$ ranges over the multi-indices, is denoted by $F(\rho)$. If $I, J$ are multi-index, $J \leq I$ will mean $j_{k} \leq i_{k}, k=1, \ldots, n$. If $P(x)=\sum a_{J} x^{J}$ is a polynomial we say that $P$ has homogeneous degree $r$ if $r=$ $\max \left\{d(I) \mid a_{I} \neq 0\right\}\left(x^{I}=x_{1}^{i_{1}} \cdots x_{n}^{i_{n}}\right)$. We use the following notation for derivatives

$$
\partial_{t}=\frac{\partial}{\partial t}, \quad D_{j}=\frac{\partial}{\partial x_{j}}, \quad D^{I}=\frac{\partial^{|I|}}{\partial x_{1}^{i_{1}} \cdots \partial_{n}^{i_{n}}} .
$$


If $r \in F(\rho), r>0$, we denote by $\wp^{r}$ the linear space of the polynomials of homogeneous degree strictly less than $r$. Finally $C$ will denote a constant, not necessarily the same in two different formulas.

\section{The spaces $\Lambda_{\alpha}^{p, q}$}

Let $D$ be a positive integer such that $D / 4 \rho_{j}$ is an integer $(j=1, \ldots, n)$. The differential operators

$$
L=\sum_{i=1}^{n} D_{j}^{D / \rho_{j}}, \quad \partial_{t}+L
$$

are easily seen to be hypoelliptic (in $R^{n}$ and $R^{n+1}$, respectively); $L$ is positive and will play the role of $-\Delta$. We also need a substitute for the Weierstrass kernel, which is given by the next proposition.

Proposition 1.1. There exists a fundamental solution $K(x, t)$ of $\partial_{t}+L$ which vanishes for all $t<0$ and such that

(i) $K \in C^{\infty}\left(R^{n+1} \backslash\{0\}\right)$ and $K(\cdot, t) \in S\left(R^{n}\right)$ for all $t>0$,

(ii) $K(-x, t)=K(x, t)$ and $K\left(r x, r^{D} t\right)=r^{-Q} K(x, t)$,

(iii) $\int_{R^{n}} K(x, t) d x=1, t>0$,

(iv) $K(\cdot, t) * K(\cdot, x)=K(\cdot, t+s), s, t>0$,

(v) if $1 \leq p \leq \infty$, then $\left\|\partial_{t}^{m} D^{I} K(\cdot, t)\right\|_{p} \leq C t^{-m d(I) / D-Q / D p^{\prime}}$, where $p^{\prime} d e$ notes the conjugate index of $p$.

Proof. Set

$$
K(x, t)= \begin{cases}\int_{R^{n}} e^{2 \pi i x \xi-t P(\xi)} d \xi & \text { if } t>0 \\ 0 & \text { if } t<0\end{cases}
$$

where $P$ is the total symbol of $L$. It is easy to verify that properties (i)-(v) hold.

For every tempered distribution $f$ we set $f(\cdot, t)=f * K(\cdot, t)$. Suppose that $k \in F(\rho), k>\alpha>0,1 \leq p \leq \infty$. If $f \in S^{\prime}\left(R^{n}\right)$ set

$$
N_{I, \alpha}^{p, q}(f)= \begin{cases}\left(\int_{0}^{\infty}\left(t^{(d(I)-\alpha) / D}\left\|D^{I} f(\cdot, t)\right\|_{p}\right)^{q} \frac{d t}{t}\right. & \text { if } 1 \leq q<\infty \\ \sup _{t>0} t^{(d(I)-\alpha) / D}\left\|D^{I} f(\cdot, t)\right\|_{p} & \text { if } q=\infty\end{cases}
$$

and

$$
N_{k, \alpha}^{p, q}(f)=\sum_{d(I) \geq k,|I| \leq \hat{k}} N_{I, \alpha}^{p, q}(f)
$$


where $\tilde{k}$ denotes the smallest integer not smaller than $k$. We define $\Lambda_{k, \alpha}^{p, q}$ as the linear space of all $f \in S^{\prime}\left(R^{n}\right)$ such that $N_{k, \alpha}^{p, q}(f)<\infty$.

(1.3) Remarks. (a) Since $N_{k, \alpha}^{p, q}(f)=0$ for every $f \in \wp^{k}, N_{k, \alpha}^{p, q}$ is not a norm on $\Lambda_{k, \alpha}^{p, q}$. Nevertheless $N_{k, \alpha}^{p, q}$ is a norm on the quotient space $\Lambda_{k, \alpha}^{p, q} / \wp^{k}$.

(b) If $\alpha<k_{1}<k_{2}$, then $\Lambda_{k_{1}, \alpha}^{p, q} \neq \Lambda_{k_{2}, \alpha}^{p, q}$; for a polynomial $P \in \wp^{k_{2}} \backslash \wp^{k_{1}}$ belongs to $\Lambda_{k_{2}, \alpha}^{p, q}$ but does not belong to $\Lambda_{k_{1}, \alpha}^{p, q}$. However $\Lambda_{k_{1}, \alpha}^{p, q} / \wp^{k_{1}}$ and $\Lambda_{k_{2}, \alpha}^{p, q} / \wp^{k_{2}}$ are equivalent spaces as will be proved in Theorem (1.5).

(c) If $1 \leq q_{1} \leq q_{2}$, then $\Lambda_{k, \alpha}^{p, q_{1}} \hookrightarrow \Lambda_{k, \alpha}^{p, q_{2}}$. The proof follows the same line as the proof of [18, Lemma 9] (see also [16, Proposition 10]).

We prepare the following lemma, which is a non-isotropic variant of $[18$, Lemma $4^{*}$.

LEMMA 1.4. Let $f$ be a tempered distribution. If $\gamma>0$ and $\partial_{t}^{j} f(x, t) \rightarrow 0$ as $t \rightarrow \infty$ for $j=0,1, \ldots, m-1$, then

$$
\left(\int_{0}^{\infty}\left(t^{\gamma}\|f(\cdot, t)\|_{p}\right)^{q} \frac{d t}{t}\right)^{1 / q} \leq C\left(\int_{0}^{\infty}\left(t^{m+\gamma}\left\|\partial_{t}^{m} f(\cdot, t)\right\|_{p}\right)^{q} \frac{d t}{t}\right)^{1 / q} .
$$

If $q=\infty$ this means

$$
\sup _{t>0} t^{\gamma}\|f(\cdot, t)\|_{p} \leq C \sup _{t>0} t^{m+\gamma}\left\|\partial_{t}^{m} f(\cdot, t)\right\|_{p}
$$

We now come to the proof of the equivalence result alluded to in Remark (1.3)(b).

THEOREM 1.5. Let $0<\alpha<k_{1}<k_{2}$.

(i) If $N_{k_{1}, \alpha}^{p, q}(f)<\infty$, then $N_{k_{2}, \alpha}^{p, q}(f) \leq N_{k_{1}, \alpha}^{p, q}(f)$.

(ii) If $N_{k_{2}, \alpha}^{p, q}(f)<\infty$, then there exists $P \in \wp^{k_{2}}$ such that $N_{k_{1}, \alpha}^{p, q}(f-P) \leq$ $C N_{k_{2}, \alpha}^{p, q}(f)$.

Proof. (i) For every tempered distribution $f$ and for every integer $s \geq \tilde{k}_{1}$, define $N_{k_{1}, \alpha, s}^{p, q}(f)=\sum_{d(I) \geq k_{1},|I| \leq s} N_{i, \alpha}^{p, q}(f)$. Clearly $N_{k_{1}, \alpha}^{p, q}(f) \leq N_{k_{1}, \alpha, s}^{p, q}(f)$. Let now $I$ be a multi-index such that $\tilde{k}_{1}<|I| \leq s$. Then there exists a multi-index $J \leq I$ such that $|J|=\tilde{k}_{1}$ and we get

$$
\begin{aligned}
\left\|D^{I} f(\cdot, t)\right\|_{p} & =\left\|D^{I-J} D^{j} f(\cdot, t)\right\|_{p} \\
& =\left\|D^{J} f(\cdot, t / 2) * D^{I-J} K(\cdot, t / 2)\right\|_{p} \\
& \leq C\left\|D^{J} f(\cdot, t / 2)\right\|_{p} t^{-(d(I)-d(J)) / D}
\end{aligned}
$$

whence $N_{l, \alpha}^{p, q}(f) \leq C N_{J, \alpha}^{p, q}(f)$. Since

$$
N_{k_{2}, \alpha}^{p, q}(f) \leq C N_{k_{1}, \alpha, \bar{k}_{2}}^{p, q}(f) \leq C N_{k_{1}, \alpha}^{p, q}(f)
$$

(i) is proved. 
(ii) Suppose first that $k_{1}$ is the antecedent of $k_{2}$ in $F(\rho)$. Then $k_{1}<k_{2} \leq$ $k_{1}+D$. Moreover $N_{k_{2}, \alpha}^{p, \infty}(f)<\infty$ by Remark (1.3)(c). Given $f \in S^{\prime}\left(R^{n}\right)$, we can write $F(x, t)=P(x, t)+R(x, t)$, where

$$
\begin{aligned}
P(x, t) & =\sum_{d(J)<k_{2}} \frac{D^{J} f(0, t)}{J !} x^{J}, \\
R(x, t) & =\sum_{\substack{d(J) \geq k_{2} \\
|J|<s}} \frac{D^{J} f(0, t)}{J !} x^{J}+s \int_{0}^{1}(1-\omega)^{s-1} \sum_{|J|=s} \frac{D^{J} f(\omega x, t)}{J !} x^{J} d \omega
\end{aligned}
$$

(here $s$ denotes any integer such that $s \geq \tilde{k}_{2}$ ). Consider the coefficients $D^{J} f(0, t)$ of the terms of maximal homogeneous degree (that is of homogeneous degree $k_{1}$ ) in $P(x, t)$. Then (suppose $u>t$ ),

$$
\begin{aligned}
\left|D^{J} f(0, u)-D^{J} f(0, t)\right| & =\left|\int_{t}^{u} \partial_{s} D^{J} f(0, s) d s\right| \\
& \leq \sum_{d(M)=k_{1}+D} \int_{t}^{u}\left|D^{M} f(0, s)\right| d s \\
& \leq \sum_{d(M)=k_{1}+D} \int_{t}^{u}\left\|D^{M} f(\cdot, s / 2)\right\|_{p}\|K(\cdot, s / 2)\|_{p^{\prime}} d s \\
& \leq N_{k_{2}, \alpha}^{p, \infty}(f) \int_{t}^{u} s^{-\left(Q / p+k_{1}+D-\alpha\right) / D} d s \\
& \leq t^{-\left(Q / p+k_{1}+D-\alpha\right) / D} N_{k_{2}, \alpha}^{p, \infty}(f)
\end{aligned}
$$

which tends to 0 as $t \rightarrow \infty$.

Let now $P_{\infty}$ be any polynomial in $\wp^{k_{2}}$ such that the coefficients of its leading terms (that is the terms of homogeneous degree $k_{1}$ ) are exactly

$$
c_{J}=\lim _{t \rightarrow \infty} \frac{D^{J} f(0, t)}{J !} .
$$

Consider

$$
\begin{aligned}
P_{\infty}(x, t) & =\left(P_{\infty} * K(\cdot, t)\right)(x)=\int_{R^{n}} \sum_{d(J)<k_{2}} c_{J}(x-y)^{J} K(y, t) d y \\
& =\sum_{d(J)<k_{2}} c_{J} \sum_{M \leq J}\left(\begin{array}{c}
J \\
M
\end{array}\right) x^{M} \int_{R^{n}}(-y)^{J-M} K(y, t) d y .
\end{aligned}
$$

Notice that $P_{\infty}(x, t)$ is in $\wp^{k_{2}}$ as a function of $x$ for every fixed $t>0$. Moreover its leading terms coincide with $c_{J}$, as is easily seen by inspection, and do not depend on $t$. Now form the difference $\left(f-P_{\infty}\right)(x, t)$. From the above discussion it follows that for every multi-index $I$ of homogeneous length $k_{1}$

$$
D^{I}\left(f-P_{\infty}\right)(x, t)=D^{I}(f(\cdot, t)-P(\cdot, t))(x)=D^{I} R(x, t) .
$$


Since

$$
\begin{aligned}
\left|D^{I} R(x, t)\right| \leq & C \sum_{\substack{d(J) \geq k_{2} \\
|J|<s}} N_{J, \alpha}^{p, \infty}(f) t^{-(Q / p+d(J)-\alpha) / D} \frac{\left|D^{I}\left(x^{J}\right)\right|}{J !} \\
& +C \int_{0}^{1}(1-\omega)^{s-1} \sum_{\substack{|J|=s \\
M+M^{\prime}}} \omega^{|M|}\left|D^{J+M} f(\omega x, t)\right| \frac{\left|D^{M^{\prime}}\left(x^{J}\right)\right|}{J !} d \omega \\
\leq & N_{k_{2}, \alpha}^{p, \infty}(f) t^{-\left(Q / p+k_{2}-\alpha\right) / D}\left\{\sum_{\substack{d(J) \geq k_{2} \\
|J|<s}}\left|D^{I}\left(x^{J}\right)\right|+\sum_{\substack{|J|=s \\
M \leq I}}\left|D^{M^{\prime}}\left(x^{J}\right)\right|\right\}
\end{aligned}
$$

we have found a polynomial $P_{\infty} \in \wp^{k_{2}}$ such that $D^{I}\left(f-P_{\infty}\right)(x, t) \rightarrow 0$ as $t \rightarrow \infty$. But, by Hölder's inequality, we also have

$$
\begin{aligned}
\left|\partial_{t} D^{I}\left(f-P_{\infty}\right)(x, t)\right| & \leq C t^{-Q / D p}\left\|\partial_{t} D^{I} f(\cdot, t / 2)\right\|_{p} \\
& \leq C t^{-Q / D p} \sum_{d(J)=D}\left\|D^{I+J} f(\cdot, t / 2)\right\|_{p} \\
& \leq C t^{-Q / D p} \sum_{d(J)=D} t^{-(d(I)+d(J)-\alpha) / D} N_{k_{2}, \alpha}^{p, \infty}(f) \rightarrow 0
\end{aligned}
$$

as $t \rightarrow \infty$. Therefore Lemma 1.4 applies and we get

$$
\begin{aligned}
N_{I, \alpha}^{p, q}\left(f-P_{\infty}\right) & \leq C\left(\int_{0}^{\infty}\left(t^{1+(d(I)-\alpha) / D}\left\|\partial_{t} D^{I}\left(f-P_{\infty}\right)(\cdot, t)\right\|_{p}\right)^{q} \frac{d t}{t}\right)^{1 / q} \\
& \leq C \sum_{d(J)=D} N_{I+J, \alpha}^{p, q}\left(f-P_{\infty}\right) \leq C N_{k_{2}, \alpha, s}^{p, q}(f) \\
& \leq C N_{k_{2}, \alpha}^{p, q}(f)
\end{aligned}
$$

for a suitable large $s$; hence (ii) is proved if $k_{1}$ is the antecedent of $k_{2}$. The general case follows by a repeated application of this result.

(1.6) Remarks. (a) Theorem 1.5 says that $\Lambda_{k_{1}, \alpha}^{p, q} / \wp^{k_{1}}$ and $\Lambda_{k_{2}, \alpha}^{p, q} / \wp^{k_{2}}$ are equivalent spaces. They are normed with any one of the (equivalent) norms $N_{k, \alpha}^{p, q}$, provided that $k>\alpha$.

(b) In the case $p=q=\infty$, a proof of part (ii) of Theorem 1.5 was given by Soardi in [15], while a related isotropic result can be found in [12]. However the techniques used in [15] are entirely different from ours. The proof is based on the isomorphism between $\Lambda_{k, \alpha}^{\infty, \infty}$ and the Morrey-Campanato spaces $L(k, \infty, a Q)$ (see [15] for the notation) and on the study of the properties of these spaces. Our proof is more direct and gives the result for all $p, q$.

(c) The following statement is a simple consequence of the definitions. 
Proposition. Suppose that $f \in \Lambda_{\alpha}^{p, q}$ and I is a multi-index such that $d(I)<$ $\alpha$. Then $D^{I} f \in \Lambda_{\alpha-d(I)}^{p, q}$.

In the following we shall drop the index $k$ and we shall write $\Lambda_{\alpha}^{p, q}$ for any one of the spaces $\Lambda_{k, \alpha}^{p, q} / \wp^{k}$.

We now introduce another norm in $\Lambda_{\alpha}^{p, q}$, which will be technically useful. Suppose that $k \in F(\rho), m$ is a positive integer not smaller than $\tilde{k} / D, 1 \leq$ $p \leq \infty, 0<\alpha<k$. For every $f \in S^{\prime}\left(R^{n}\right)$ we set

$$
N_{k, \alpha}^{* m, p, q}(f)= \begin{cases}\left(\int_{0}^{\infty}\left(t^{m-\alpha / D}\left\|\partial_{t}^{m} f(\cdot, t)\right\|_{p}\right)^{q} \frac{d t}{t}\right)^{1 / q} & \text { if } 1 \leq q<\infty, \\ \sup _{t>0} t^{m-\alpha / D}\left\|\partial_{t}^{m} f(\cdot, t)\right\|_{p} & \text { if } q=\infty .\end{cases}
$$

In the isotropic non-homogeneous case, norms of this type have been extensively studied by Taibleson [18] and Flett [2].

THEOREM 1.7. In $\Lambda_{\alpha}^{p, q}, N_{k, \alpha}^{p, q}$ and $N_{k, \alpha}^{* m, p, q}$ are equivalent norms.

Proof. We first prove that $N_{k, \alpha}^{* m, p, q}(f) \leq C N_{k, \alpha}^{p, q}(f)$. Since

$$
\partial_{t}^{m} f(\cdot, t)=(-1)^{m} L^{m} f(\cdot, t)
$$

we have $N_{k, \alpha}^{* m, p, q}(f) \leq \sum_{d(I)=m D} N_{I, \alpha}^{p, q}(f)$. If $I$ is a multi-index such that $|I| \leq$ $\tilde{k}_{1}$, then $N_{I, \alpha}^{p, q}(f) \leq N_{k, \alpha}^{p, q}(f)$. Let now $I$ be a multi-index such that $|I|>\tilde{k}_{1}$. Choose $J \leq I$ such that $|J|=\tilde{k}$. Then $d(J) \geq k$. Therefore, by Proposition 1.1(v),

$$
\begin{aligned}
N_{I, \alpha}^{p, q}(f) & =\left(\int_{0}^{\infty}\left(t^{(d(I)-\alpha) / D}\left\|D^{I-J} D^{J} f(\cdot, t)\right\|_{p}\right)^{q} \frac{d t}{t}\right)^{1 / q} \\
& \leq C N_{J, \alpha}^{p, q}(f) \leq C N_{k, \alpha}^{p, q}(f) .
\end{aligned}
$$

Conversely we first notice that $N_{k, \alpha}^{p, \infty}(f)<\infty$, by Remark (1.3)(c). Write $p^{\prime}$ for the conjugate index of $p$. Then for every $s \geq 0$,

$$
\begin{aligned}
\left\|\partial_{t}^{s} D^{I} f(\cdot, t)\right\|_{\infty} & \leq\left\|D^{I} f(\cdot, t / 2)\right\|_{p}\left\|\partial_{t}^{s} K(\cdot, t / 2)\right\|_{p^{\prime}} \\
& \leq C t^{-(d(I)-\alpha) / D-s-Q / p D} N_{k, \alpha}^{p, \infty}(f) .
\end{aligned}
$$

Therefore Lemma 1.4 applies. Setting $\gamma=(d(I)-\alpha) / D$ we have

$$
\begin{array}{rl}
\left(\int_{0}^{\infty}\left(t^{\gamma}\left\|D^{I} f(\cdot, t)\right\|_{p}\right)^{q} \frac{d t}{t}\right)^{1 / q} & C\left(\int_{0}^{\infty}\left(t^{m+\gamma}\left\|\partial_{t}^{m} D^{I} f(\cdot, t)\right\|_{p}\right)^{q} \frac{d t}{t}\right)^{1 / q} \\
& \leq C N_{k \alpha}^{* m, p, q}(f),
\end{array}
$$

which gives the desired result. 
Remark. For every $\gamma>0$, set

$$
G_{\gamma}(x)=\Gamma(\gamma / D)^{-1} \int_{0}^{\infty} t^{\gamma / D-1} e^{-t} K(x, t) d t .
$$

It is not hard to show that $\hat{G}_{y}(\xi)=(1+P(\xi))^{-\gamma / D}$, where $P$ is the total symbol of the operator $L$. Now define the Bessel potential operators of order $\gamma$ as follows

$$
(\hat{\mathscr{J}} f)(\xi)=(1+P(\xi))^{-\gamma / D} \hat{f}(\xi)
$$

Note that $\mathscr{I}_{\gamma}$ coincide with the quasi-homogeneous Bessel potential operators considered in [1]; the explicit knowledge of the kernel $G_{\gamma}$ greatly simplifies the treatment in [1]. Our definition of Lipschitz spaces makes it easy to show that, for example

$$
G_{\gamma} \in \Lambda_{\gamma-Q / p}^{p^{\prime}, \infty}, \quad(\gamma>Q / p) .
$$

In fact, if $d(I)>\gamma-Q / p$

$$
\begin{aligned}
\left\|D^{I} G_{\gamma}(\cdot, t)\right\|_{p^{\prime}} & \leq C \int_{0}^{\infty} r^{\gamma / D} e^{-r}\left\|D^{I} K(\cdot, t+r)\right\|_{p^{\prime}} \frac{d r}{r} \\
& \leq C \int_{0}^{\infty} r^{\gamma / D} e^{-r}(r+t)^{-(d(I)+Q / p) / D} \frac{d r}{r} \\
& \leq C t^{-(d(I)-(\gamma-Q / p)) / D} .
\end{aligned}
$$

The last inequality follows integrating by parts. The techniques previously developed permit us to prove without difficulty the following result (see also [18] and [8]).

Theorem. Let $g \in \Lambda_{\gamma}^{1, \infty}$. Then there exists a positive constant $C$ such that for every compactly supported $f \in \Lambda_{\alpha}^{p, q}$ we have

$$
N_{k_{1}+k_{2}, \alpha+\gamma}^{p, q}(f * g) \leq C N_{k_{1}, \alpha}^{p, q}(f)
$$

where $k_{1}, k_{2} \in F(\rho), k_{1}>\alpha, k_{2}>\gamma$.

A simple consequence of the above theorem is the following

COROLLARY. $\mathscr{J}_{y}$ maps the functions $f \in \Lambda_{\alpha}^{p, q}$ with compact support continuously into $\Lambda_{\alpha+\gamma}^{p, q}$.

We do not pursue the matter any further, but it is clear that many results concerning the theory of the Bessel and also of the Riesz potentials (see 
[10] for some mapping properties of non-isotropic Riesz potentials) can be transferred without difficulty to the non-isotropic setting.

\section{The main result}

Let $k \in F(\rho)$ and denote by $N(k)$ the number of distinct elements of $F(\rho)$ smaller than $k$. Then there exists exactly one $(N+1)$-tuple $c_{0}=1, c_{1}, \ldots, c_{n}$ satisfying

$$
\sum_{j=0}^{N(k)} c_{j} j^{d(I)}=0 \text { if and only if } 0 \leq d(I)<k
$$

(see [15] for details).

For every smooth function $\phi$ and every $k \in F(\rho)$ we define the nonisotropic $k$ th order difference centered at $x$ as

$$
\Delta_{h}^{k} \phi(x)=\sum_{j=0}^{N(k)} c_{j} \phi(x+j h), \quad x, h \in R^{n}
$$

(recall that $j h$ stands for $A_{j} h$ ). For every distribution $f$ we define

$$
\left\langle\Delta_{h}^{k} f, \phi\right\rangle=\left\langle f, \Delta_{-h}^{k} \phi\right\rangle
$$

for every test function $\phi$. In the following we shall write $N$ instead of $N(k)$.

Suppose that $k \in F(\rho), 0<\alpha<k, 1 \leq p \leq \infty$. For every locally integrable tempered distribution $f$ (locally bounded if $p=\infty$ ) we set

$$
M_{k, \alpha}^{p, q}(f)= \begin{cases}\left(\int_{R^{n}}\left\|\Delta_{h}^{k} f\right\|_{p}^{q}|h|^{-\alpha q-Q} d h\right)^{1 / q} & \text { if } 1 \leq q<\infty, \\ \sup _{h \neq 0} \frac{\left\|\Delta_{h}^{k} f\right\|_{p}}{|h|^{\alpha}} & \text { if } q=\infty .\end{cases}
$$

We now prove that if $f \in \Lambda_{\alpha}^{p, q}$, then $f$ is a locally integrable (locally bounded if $p=\infty$ ) function.

Lemma 2.2. If $f \in \Lambda_{\alpha}^{p, q}$, then $f=g+h$ where $g \in L^{p}\left(R^{n}\right)$ and $h$ is a slowly increasing $C^{\infty}$ function.

Proof. By Remark (1.3)(c), $f \in \Lambda_{\alpha}^{p, \infty}$. We first show that if $f \in \Lambda_{\alpha}^{p, \infty}$ then $\left\|\Delta_{h}^{k} f\right\|_{p} \leq C|h|^{\alpha}$ for every $h \in R^{n}$. 
Suppose that $t \geq|h|^{D}$. Then by Taylor's formula

$$
\begin{aligned}
\left\|\Delta_{h}^{k} f(\cdot, t)\right\|_{p} & \leq \sum_{\substack{d(I)>k \\
|I| \leq k}}|h|^{d(I)}\left\|D^{I} f(\cdot, t)\right\|_{p} \\
& =C|h|^{\alpha} \sum_{\substack{d(I) \geq k \\
|I| \leq k}}\left(\frac{|h|}{t^{1 / D}}\right)^{d(I)-\alpha} t^{(d(I)-\alpha) / D}\left\|D^{I} f(\cdot, t)\right\|_{p} \\
& \leq C|h|^{\alpha} N_{k, \alpha}^{p, \infty}(f) .
\end{aligned}
$$

Let now $0<t<t_{0}=|h|^{D}$. By Taylor's formula

$$
\left|\Delta_{h}^{k} f(x, t)\right| \leq C\left\{\sum_{j=0}^{m-1} t_{0}^{j}\left|\partial_{t}^{j} \Delta_{h}^{k} f\left(x, 2 t_{0}\right)\right|+\int_{t}^{2 t_{0}} u^{m-1}\left|\partial_{u}^{m} \Delta_{h}^{k} f(x, u)\right| d u\right\}
$$

from which we get

$$
\begin{aligned}
\sup _{0<t<t_{0}}\left\|\Delta_{h}^{k} f(\cdot, t)\right\|_{p} & \leq C\left\{\left\|\Delta_{h}^{k} f\left(\cdot, t_{0}\right)\right\|_{p}+t_{0}^{\alpha / D} N_{k, \alpha}^{p, \infty}(f)\right\} \\
& \leq C|h|^{\alpha} N_{k, \alpha}^{p, \infty}(f) .
\end{aligned}
$$

Therefore if $p>1, \Delta_{h}^{k} f \in L^{p}$ by a standard weak*-compactness argument (see for example the proof of [17, Theorem 2.5]). The case $p=1$ is more delicate and we only give a sketch of the proof. By $(2.3)\left\|\partial_{t}^{s} g(\cdot, t)\right\|_{\infty} \rightarrow 0$ as $t \rightarrow \infty$ for every integer $s \geq 0$. Therefore Lemma (1.4) can be applied. Suppose first that $0<\alpha / D<1$ : then, by Theorem 1.7

$$
\left\|\partial_{t} g(\cdot, t)\right\|_{1} \leq C t^{\alpha / D-1} N_{k, \alpha}^{1, \infty}(f) .
$$

Let now $\alpha / D \geq 1$. Then

$$
\begin{gathered}
\sup _{0<t<1} t^{m-1 / 2}\left\|\partial_{t}^{m} g(\cdot, t)\right\|_{1} \leq C N_{k, \alpha}^{1, \infty}(f), \\
\sup _{t>1} t^{m-1 / 2}\left\|\partial_{t}^{m} g(\cdot, t)\right\|_{1} \leq C \sup _{t>1} t^{-1 / 2}\|g(\cdot, t)\|_{1} \leq C N_{k, \alpha}^{1, \infty}(f)|h|^{\alpha} .
\end{gathered}
$$

Gathering terms we get

$$
\sup _{t>0} t^{m-1 / 2}\left\|\partial_{t}^{m} g(\cdot, t)\right\|_{1} \leq C,
$$

which implies

$$
\left\|\partial_{t} g(\cdot, t)\right\|_{1} \leq C t^{1-1 / 2} .
$$

Let $\beta$ denote $\alpha / D$ if $0<\alpha / D<1$ and $1 / 2$ if $\alpha / D \geq 1$. Then

$$
\left\|g\left(\cdot, t_{1}\right)-g\left(\cdot, t_{2}\right)\right\|_{1} \leq \int_{t_{1}}^{t_{2}}\left\|\partial_{t} g(\cdot, t)\right\|_{1} d t \leq \int_{t_{1}}^{t_{2}} t^{\beta-1} d t \rightarrow 0
$$


as $t_{1}, t_{2} \rightarrow 0$ and the case $p-1$ follows. Now, by homogeneity

$$
\begin{aligned}
\int_{R^{n}} \Delta_{h}^{k} f(x, s) K(h, t) d h & =\sum_{j=1}^{n} c_{j} \int_{R^{n}} f(x+h, s) K\left(h, j^{D} t\right) d h-f(x, s) \\
& =\sum_{j=1}^{n} c_{j} f\left(x, s+j^{D} t\right)-f(x, s) .
\end{aligned}
$$

Set $\tilde{f}(x)=\sum_{j=1}^{n} c_{j} f\left(x, j^{D} t\right)-\int_{R^{n}} \Delta_{h}^{k} f(x) K(h, t) d h$. Then $\tilde{f}$ is the sum of a slowly increasing function and of an $L^{p}$ function, since

$$
\left\|\int_{R^{n}} \Delta_{h}^{k} f(x) K(h, t) d h\right\|_{L^{p}(d x)} \leq C \int_{R^{n}}|h|^{\alpha}|K(h, t)| d h .
$$

We only need to show that $\tilde{f}$ coincides with $f$. This follows from the easily verified fact that $\tilde{f}(x, s)=f(x, s)$

REMARK. The idea of considering the expression $\int \Delta_{h}^{k} f(x, s) K(h, t) d t$ is taken from [7], where a similar but isotropic case is treated.

We are now ready to prove our main result.

THEOREM 2.4. (i) There is a constant $C>0$ such that for every $f \in \Lambda_{a}^{p, q}$, we have $M_{k, \alpha}^{p, q}(f) \leq C N_{k, \alpha}^{p, q}(f)$.

(ii) There is a constant $C>0$ such that for every locally integrable function $f$ (locally bounded if $p=\infty$ ) satisfying $M_{k, \alpha}^{p, q}(f)<\infty$, we have $N_{k, \alpha}^{p, q}(f) \leq$ $C M_{k, \alpha}^{p, q}(f)$.

Proof. (i) Suppose that $f \in \Lambda_{\alpha}^{p, q}$ and let $k \in F(\rho)$ be $>\alpha$. Then by Lemma $2.2 f$ is a locally integrable function. Let $S^{n-1}$ denote the unit sphere in $R^{n}$ and let $h=r \xi$, the non-isotropic polar decomposition of $h$. Set

$$
\begin{aligned}
& \Omega_{p, q}^{1}(r)=\int_{S^{n-1}}\left\|\Delta_{r \xi}^{k}(\cdot, t)\right\|_{p}^{q} d \xi, \\
& \Omega_{p, q}^{2}(r)=\int_{S^{n-1}}\left\|\Delta_{r \xi}^{k}(f-f(\cdot, t))\right\|_{p}^{q} d \xi .
\end{aligned}
$$

By Taylor's formula

$$
\begin{aligned}
\Omega_{p, q}^{1}(r) & \leq C \int_{S^{n-1}}\left(\sum_{\substack{d(I) \geq k \\
|I| \leq \hat{k}}} r^{d(I)}\left\|D^{I} f(\cdot, t)\right\|_{p}\right)^{q} d \xi \\
& \leq C\left(\sum_{\substack{d(I) \geq k \\
|I| \leq \tilde{k}}} r^{d(I)}\left\|D^{I} f(\cdot, t)\right\|_{p}\right)^{q} .
\end{aligned}
$$


Since $K(\cdot, 1)$ is a Schwartz function, its least decreasing majorant is integrable so that (see [16, page 62]) $\Delta_{r \xi}^{k}(\cdot, t) \rightarrow \Delta_{r \xi}^{k} f$ for almost every $x \in R^{n}$. Thus, by Taylor's formula with respect to the real variable $t$,

$\left\|\Delta_{r \xi}^{k}(f-f(\cdot, t))\right\|_{p} \leq C\left(\sum_{j=1}^{m-1} t^{j}\left\|\partial_{t}^{j} \Delta_{r \xi}^{k} f(\cdot, t)\right\|_{p}+\int_{0}^{t} u^{m-1}\left\|\partial_{u}^{m} \Delta_{r \xi}^{k} f(\cdot, u)\right\|_{p} d u\right)$

Here $m$ denotes any integer greater than $\tilde{k} / D$. Therefore,

$$
\begin{aligned}
\Omega_{p, q}^{2}(r) \leq C \int_{s^{n-1}} & \left(\sum_{j=1}^{m-1} t^{j}\left\|\partial_{t}^{j} \Delta_{r \xi}^{k}(\cdot, t)\right\|_{p}\right. \\
& \left.\quad+\int_{0}^{t} u^{m-1}\left\|\partial_{u}^{m} \Delta_{r \xi}^{k} f(\cdot, u)\right\|_{p} d u\right)^{q} d \xi .
\end{aligned}
$$

Using polar coordinates and Minkowski’s inequality, we get

$$
\begin{aligned}
M_{k, \alpha}^{p, q}(f) & =\left(\int_{0}^{\infty} r^{-\alpha q-1}\left(\int_{s^{n-1}}\left\|\Delta_{r \xi}^{k} f\right\|_{p}^{q} d \xi\right) d r\right)^{1 / q} \\
& \leq\left(\int_{0}^{\infty} r^{-\alpha q-1}\left(\left(\Omega_{p, q}^{1}(R)\right)^{1 / q}+\left(\Omega_{p, q}^{2}(r)\right)^{1 / q}\right)^{q} d r\right)^{1 / q} \\
& \leq \sum_{j=1}^{2}\left(\int_{0}^{\infty} r^{-\alpha q-1} \Omega_{p, q}^{j}(r) d r\right)^{1 / q}=I_{1}+I_{2} .
\end{aligned}
$$

By (2.5)

$$
\begin{aligned}
I_{1} & \leq C\left(\int_{0}^{\infty} r^{-\alpha q-1}\left(\sum_{\substack{d(I) \geq k \\
|I| \leq \tilde{k}}} r^{d(I)}\left\|D^{I} f(\cdot, t)\right\|_{p}\right)^{q} d r\right)^{1 / q} \\
& \leq C \sum_{\substack{d(I) \geq k \\
|I| \leq \tilde{k}}}\left(\int_{0}^{\infty}\left(r^{d(I)-\alpha}\left\|D^{I} f(\cdot, t)\right\|_{p}\right)^{q} \frac{d r}{r}\right)^{1 / q} .
\end{aligned}
$$

Setting $t=r^{D}$ and changing variables we get $I_{1} \leq N_{k, \alpha}^{p, q}(f)$. Now, by Proposition $1.1(\mathrm{v}), t^{j}\left\|\partial_{t}^{j} \Delta_{r \xi}^{k} f(\cdot, t)\right\|_{p} \leq C\left\|\Delta_{r \xi}^{k} f(\cdot, t)\right\|_{p}$. Therefore, taking (2.6) into 
account, we have

$$
\begin{aligned}
I_{2} & \leq C\left\{\int _ { 0 } ^ { \infty } r ^ { - \alpha q - 1 } \int _ { S ^ { n - 1 } } \left(\left\|\Delta_{r \xi}^{k} f(\cdot, t)\right\|_{p}\right.\right. \\
& \left.\left.+\int_{0}^{t} u^{m-1}\left\|\partial_{u}^{m} \Delta_{r \xi}^{k} f(\cdot, u)\right\|_{p} d u\right)^{q} d r d \xi\right\}^{1 / q} \\
& \leq C\left\{\int _ { 0 } ^ { \infty } r ^ { - \alpha q - 1 } \left(\left(\int_{s^{n-1}}\left\|\Delta_{r \xi}^{k} f\right\|_{p}^{q} d \xi\right)^{1 / q}\right.\right. \\
& \left.\left.+\left(\int_{s^{n-1}}\left(\int_{0}^{t} u^{m-1}\left\|\partial_{u}^{m} \Delta_{r \xi}^{k} f(\cdot, u)\right\|_{p} d u\right)^{q} d \xi\right)^{1 / q}\right)^{q} d r\right\}^{1 / q} \\
& \leq C\left\{I_{1}+\left(\int_{0}^{\infty} r^{-\alpha q-1}\left(\int_{S^{n-1}} \int_{0}^{t} u^{m-1}\left\|\partial_{u}^{m} \Delta_{r \xi}^{k} f(\cdot, u)\right\|_{p} d u\right)^{q} d \xi\right)^{1 / q} d r\right\}^{1 / q} \\
& =C\left(I_{1}+I_{3}\right) .
\end{aligned}
$$

An estimate of $I_{1}$ has been given above. Using the fact that $\left\|\partial_{u}^{m} \Delta_{r \xi}^{k} f(\cdot, u)\right\|_{p} \leq$ $\left\|\partial_{u}^{m} f(\cdot, u)\right\|_{p}$, setting $t=r^{D}$, changing variables and taking Theorem 1.7 into account, we have

$$
\begin{aligned}
I_{3} & \leq C\left(\int_{0}^{\infty} r^{-\alpha q / D-1}\left(\int_{0}^{r} u^{m-1}\left\|\partial_{u}^{m} f(\cdot, u)\right\|_{p} d u\right)^{q} d r\right)^{1 / q} \\
& \leq C\left(\int_{0}^{\infty}\left(u^{m-\alpha / D}\left\|\partial_{u}^{m} f(\cdot, u)\right\|_{p}\right)^{q} \frac{d u}{u}\right)^{1 / q} \\
& \leq C N_{k, \alpha}^{p, q}(f),
\end{aligned}
$$

which gives (i).

(ii) Suppose that $\phi$ is a smooth function with compact support contained in the unit centered at the origin and $\int \phi=1$. For every integer $r$ set

$$
p_{r}=\sum_{j=1}^{N} c_{j} \phi_{j 2^{-}}
$$

where $c_{j}$ 's and $N$ are the same as in (2.1). Set

$$
P_{r}=p_{r}-p_{r-1}, \quad Q_{r}=p_{r}+p_{r-1} .
$$

Then if $f \in S^{\prime}\left(R^{n}\right)$ is locally integrable and $M_{k, \alpha}^{p, q}(f)<\infty$ we have

$$
D^{I} f(\cdot, t)=\sum_{r=-\infty}^{\infty} D^{I} f(\cdot, t) * P_{r} * Q_{r}
$$

for every $I$ such that $d(I) \geq k$. Since

$$
f * P_{r}(x)=\int_{R^{n}} \Delta_{2^{-r} y}^{k} f(x) g(-y) d y-\int_{R^{n}} \Delta_{2^{-r+1} y}^{k} f(x) g(-y) d y
$$


we have

$$
\left\|f * P_{r}\right\|_{p} \leq C 2^{r Q_{\gamma}}(r)
$$

where $\gamma(r)=\int_{|y| \leq 2^{-r}}\left\|\Delta_{y}^{k} f\right\|_{p} d y$. Moreover

$$
\left\|D^{I} Q_{r}\right\|_{1} \leq C \min \left(1,2^{r d(I)}\right) .
$$

Choose an integer $s$ such that $2^{-s+1}<t^{1 / D} \leq 2^{-s}$. Then, by (2.7) and (2.8)

$$
\begin{aligned}
\left\|D^{I} f(\cdot, t)\right\| \leq & \sum_{-\infty}^{s-1}\|K(\cdot, t)\|_{1}\left\|f * P_{r}\right\|_{p}\left\|D^{I} Q_{r}\right\|_{1} \\
& +\sum_{s}^{\infty}\left\|D^{I} K(\cdot, t)\right\|_{1}\left\|f * P_{r}\right\|_{p} \|_{Q_{r} \|_{1}} \\
\leq & C\left(\sum_{-\infty}^{s-1} 2^{r(d(I)+Q)} \gamma(r)+t^{-d(I) / D} \sum_{s}^{\infty} 2^{r Q} \gamma(r)\right) \\
\leq & C\left(\int_{t^{1 / D}}^{\infty} u^{-d(I)-Q-1} \gamma(u) d u\right. \\
& +t^{-d(I) / D} \int_{0}^{t^{1 / D}} u^{\left.-1-Q_{\gamma}(u) d u\right) .}
\end{aligned}
$$

Therefore

$$
\begin{aligned}
& N_{k, \alpha}^{p, q}(f) \leq C\left\{\left(\int_{0}^{\infty}\left(t^{(d(I)-\alpha) / D} \int_{t^{1 / D}}^{\infty} u^{-d(I)-Q-1} \gamma(u) d u\right)^{q} \frac{d t}{t}\right)^{1 / q}\right.\left.+\left(\int_{0}^{\infty}\left(t^{-\alpha / D} \int_{0}^{t^{1 / D}} u^{-1-Q} \gamma(u) d u\right)^{q} \frac{d t}{t}\right)^{1 / q}\right\} \\
&=J_{1}+J_{2} .
\end{aligned}
$$

Changing variables, using the Hardy-Littlewood inequalities and setting $y=$ $r \xi$, we get

$$
\begin{aligned}
J_{1} & \leq C\left(\int_{0}^{\infty} u^{-(Q+\alpha) q-1}\left(\int_{0}^{u}\left(\int_{S^{n-1}}\left\|\Delta_{r \xi}^{k} f\right\|_{p} d \xi\right) r^{Q-1} d r\right)^{q} d u\right)^{1 / q} \\
& \leq C\left(\int_{0}^{\infty}\left(\Omega(r) r^{Q}\right)^{q} r^{-(Q+\alpha) q-1} d r\right)^{1 / q} \\
& =C\left(\int_{0}^{\infty} r^{-\alpha q-1}\left(\int_{S^{n-1}}\left\|\Delta_{r \xi}^{k} f\right\|_{p} d \xi\right)^{q} d r\right)^{1 / q} \\
& \leq C M_{k, \alpha}^{p, q}(f) .
\end{aligned}
$$

Now $J_{2}$ can be estimated in the same way as $J_{1}$ and (ii) follows. 
Remarks. (a) The technique used in (ii) of the proof of Theorem 2.4 is a refinement of some ideas of I. Inglis (see [10]; and [5], [15] for related matters).

(b) Sometimes it is more convenient to use moduli of smoothness instead of difference operators. For every $f \in S^{\prime}\left(R^{n}\right)$ which is locally integrable (locally bounded if $p=\infty$ ) set

$$
L_{k, \alpha}^{p, q}(f)= \begin{cases}\left(\int_{0}^{\infty}\left(t^{-\alpha} \omega_{k}\left(t, f, L^{p}\right)\right)^{q} \frac{d t}{t}\right)^{1 / q} & \text { if } 1 \leq q<\infty, \\ \sup _{t>0} t^{-\alpha} \omega_{k}\left(f, t, L^{p}\right) & \text { if } q=\infty,\end{cases}
$$

(here $\left.\omega_{k}\left(t, f, L^{p}\right)=\sup _{0<|h| \leq t}\left\|\Delta_{h}^{k} f\right\|_{p}\right)$. Clearly $M_{k, \alpha}^{p, q}(f) \leq L_{k, \alpha}^{p, q}$. In fact these two norms are equivalent in $\lambda_{\alpha}^{p, q}$. A simple proof can be accomplished by using the following inequality

$$
\omega_{k}\left(t, f, L^{p}\right) \leq C\left(\left\|f-g_{t}\right\|_{p}+\sum_{\substack{d(I) \geq k \\|I| \leq \tilde{k}}} t^{d(I)}\left\|D^{I} g_{t}\right\|_{p}\right) .
$$

We refer the reader to [15] for the notation used here and the proof of the above inequality.

\section{References}

[1] M. Cotlar and C. Sadosky, 'On quasi-homogeneous Bessel potential operators,' Proc. Sympos. Pure Math. 10 (1966), 275-287.

[2] T. M. Flett, 'Temperatures, Bessel potential and Lipschitz spaces,' Proc. London Math. Soc. 22 (1971), 385-207.

[3] G. B. Folland, 'Subelliptic estimates and function spaces on nilpotent Lie groups,' Ark. Math. 13 (1975), 161-207.

[4] G. B. Folland and E. M. Stein, Hardy spaces on homogeneous groups, (Math. Notes 28, Princeton, 1982).

[5] G. I. Gaudry and R. Pini, 'Bernstein's theorem for compact connected Lie groups,' Math. Proc. Cambridge Philos. Soc. 99 (1986), 297-305.

[6] S. Giulini, 'Approximation and Besov spaces on stratified groups,' Proc. Amer. Math. Soc. 96 (1986), 569-578.

[7] H. Greenwald, 'On the theory of homogeneous Lipschitz spaces and Campanato spaces,' Pacific J. Math. 106 (1983), 87-93.

[8] C. Herz, 'Lipschitz spaces and Bernstein's theorem on absolutely convergent Fourier transform,' J. Math. Mech. 18 (1968), 283-323.

[9] I. R. Inglis, 'Bernstein's theorem and the Fourier algebra of the Heisenberg group,' Boll. Un. Mat. Ital. (VI) 2-A (1983), 39-46.

[10] I. R. Inglis, 'Weak and strong mapping properties of translation invariant operators,' Boll. Un. Mat. Ital. (VI) 1-B (1982), 523-533.

[11] S. Janson, 'Generalizations of Lipschitz spaces and an application to Hardy spaces and bounded mean oscillation,' Duke Math. J. 47 (1980), 959-982. 
[12] S. Janson, M. H. Taibleson and G. Weiss, 'Elementary characterizations of the MorreyCompanato spaces,' Harmonic Analysis, edited by G. Mauceri, F. Ricci and G. Weiss, pp. 101-104 (Lecture Notes in Mathematics 992, Springer-Verlag, 1983).

[13] R. Johnson, 'Temperatures, Riesz potentials and the Lipschitz spaces of Herz,' Proc. London Math. Soc. 27 (1973), 290-316.

[14] S. G. Krantz, 'Geometric Lipschitz spaces and applications to complex function theory and nilpotent groups,' J. Funct. Anal. 34 (1979), 456-471.

[15] P. M. Soardi, 'On non-isotropic Lipschitz spaces,' Harmonic Analysis, edited by G. Mauceri, F. Ricci and G. Weiss, pp. 115-138 (Lecture Notes in Mathematics 992, SpringerVerlag, 1983).

[16] E. M. Stein, Singular Integrals and Differentiability Properties of Functions, (Princeton University Press, 1970).

[17] E. M. Stein and G. Weiss, Introduction to Fourier Analysis on Euclidean Spaces, (Princeton University Press, 1971).

[18] M. H. Taibleson, 'On the theory of Lipschitz spaces of distributions on Euclidean $n$-spaces, I,' J. Math. Mech. 13 (1964), 407-480.

[19] M. H. Taibleson, 'On the theory of Lipschitz spaces of distributions on Euclidean $n$-spaces, II,' J. Math. Mech. 14 (1965), 821-839.

[20] A. Torchinski, 'Singular integrals in the space $\Lambda(B, X)$,' Studia Math. 48 (1973), 165-189.

\author{
Dipartimento di Matematica \\ dell'Università di Trento \\ 58050 Povo (TN) \\ Italy
}

